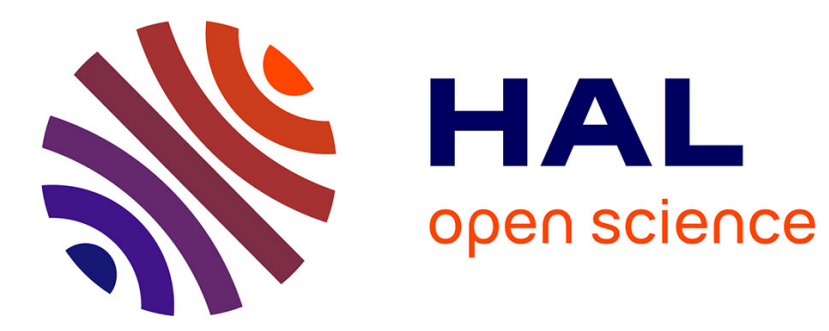

\title{
Les enjeux actuels de l'évolution des métiers de la diplomatie
}

Marc Loriol

\section{To cite this version:}

Marc Loriol. Les enjeux actuels de l'évolution des métiers de la diplomatie. Les Cahiers Sirice / Irice , 2009, 3, pp.81-102. halshs-00400014

\section{HAL Id: halshs-00400014 https://shs.hal.science/halshs-00400014}

Submitted on 29 Jun 2009

HAL is a multi-disciplinary open access archive for the deposit and dissemination of scientific research documents, whether they are published or not. The documents may come from teaching and research institutions in France or abroad, or from public or private research centers.
L'archive ouverte pluridisciplinaire HAL, est destinée au dépôt et à la diffusion de documents scientifiques de niveau recherche, publiés ou non, émanant des établissements d'enseignement et de recherche français ou étrangers, des laboratoires publics ou privés. 


\section{Les enjeux actuels de l'évolution des métiers de la diplomatie}

Les Cahiers IRICE, $\mathrm{n}^{\circ} 3$, 2009, «Diplomaties en renouvellement » (sous la direction de Laurence Badel et Stanislas Jeannesson), pp. 81-102.

Par Marc Loriol

Dans le cadre de la LOLF (Loi organique relative aux lois de finances), de la RGPP (Révision générale des politiques publiques) ou de la modernisation de l'Etat, tout un ensemble de mesures visent à rationaliser, voire à réduire, l'action de 1'Etat à l'étranger et à optimiser le travail des diplomates. Dans le même temps, certains auteurs vont parfois jusqu'à écrire qu'avec la forte croissance des rencontres entre responsables politiques de différents pays, la montée en puissance de nouveaux acteurs (ONG, entreprises, collectivités locales, organisations internationales...) - la diplomatie " polylatérale ${ }^{1}{ }^{-}$, les nouveaux moyens de communication (Internet, visioconférences...), le travail des diplomates perdrait de son importance, les relations internationales pouvant être conduites sans leur concours. Cette crainte n'est pas nouvelle. Dans les Confessions d'un vieux diplomate, le comte de SaintAulaire, écrivait ainsi en 1953 : «La diplomatie que j'ai connue, la diplomatie des diplomates n'existe plus $»^{2}$. Trente ans plus tard, Albert Chambon notait de même : « Dès que 1'on parle, aujourd'hui, du métier de diplomate, la question est posée de savoir si au $20^{\mathrm{e}}$ siècle, ce fonctionnaire n'a pas perdu de son importance par rapport au rôle qu'il pouvait avoir dans les siècles passés $)^{3}$. Ces réflexions sur la perte d'utilité des diplomates sont toutefois contredites par l'observation du travail quotidien des agents du ministère des Affaires étrangères et européennes (MAEE). Comme le faisait remarquer un diplomate américain à un chercheur : «Si la diplomatie est moins importante aujourd'hui qu'auparavant, comment se fait-il que je sois toujours aussi débordé de travail ? ». Il semble en effet y avoir un hiatus important entre les théories sur la diplomatie, qui la présentent comme une pratique dépassée, et l'observation que l'on peut faire du travail quotidien des diplomates.

Les différentes facettes de l'activité diplomatique peuvent se résumer en quatre verbes, souvent utilisés par les diplomates eux-mêmes lorsqu'ils parlent de leur travail : représenter, informer, négocier, organiser. Prises séparément, ces activités ne fondent pas un métier spécifique, mais c'est leur combinaison, la présence constante de ces quatre dimensions qui donne une tonalité particulière au travail diplomatique. A partir d'une enquête en cours sur le travail des diplomates ${ }^{5}$, je voudrais discuter et relativiser certains points de ces discours sur le changement. Le rôle des diplomates à l'époque des déplacements fréquents des responsables politiques et de la société de l'information reste important et finalement peu différent, dans sa logique, de ce qui a pu être décrit par le passé. Il n'est pas possible ici de reprendre une à une

\footnotetext{
${ }^{1}$ Ce néologisme forgé par Kofi Annan entend souligner le fait que dans les négociations multilatérales entre pays interviendraient un nombre croisant d'acteurs non-étatiques (ONG, multinationales, collectivités locales, etc.).

${ }^{2}$ Auguste-Félix Charles de Beaupoil Comte de Saint-Aulaire, Confessions d'un vieux diplomate, Paris, Flammarion, 1953

${ }^{3}$ Albert Chambon, Mais que font donc ces diplomates entre deux cocktails ?, Paris, A. Pedone, 1983.

${ }^{4}$ Cité dans Iver B. Neumann, « To be a Diplomat », International Studies Perspectives, 2005, n ${ }^{\circ}$, p. $72-93$.

${ }^{5}$ Recherche menée par Marc Loriol (responsable), Françoise Piotet, Valérie Boussard, David Delfolie et Vincent Porteret. Un échantillon d'une quarantaine d'agents de catégorie A de l'administration centrale à Paris a été tiré au sort dans les différentes directions afin de réaliser des entretiens approfondis. Surtout, sept postes (quatre bilatéraux dont les consulats et trois multilatéraux), trois directions parisiennes et les services nantais ont fait l'objet d'études plus approfondies (entretiens auprès de différentes catégories de personnel et observations). Afin de pouvoir observer librement les différentes phases de travail (réunion, préparation des visites, travail consulaires, etc.) et selon les règles déontologiques en vigueur dans la recherche sociologique, un anonymat total a été garanti pour toutes les personnes qui ont bien voulu nous recevoir
} 
les différentes facettes du métier. Nous avons donc choisi de centrer l'analyse sur deux aspects du travail, particulièrement pertinents par rapport à la question de l'éventuelle évolution du rôle des diplomates: d'une part, l'augmentation des déplacements des responsables politiques et la place des diplomates dans la préparation des voyages officiels ; d'autre part, l'impact des technologies de l'information et de la communication sur le travail d'information.

\section{Les déplacements des responsables politiques peuvent-ils se substituer au travail des diplomates?}

Une activité particulière regroupe et met en œuvre les quatre grands types d'activités qui structurent le travail diplomatique : 1'organisation et la gestion des visites officielles au cours desquelles sont mises en scène les relations bilatérales, finalisées certaines négociations et qui donnent lieu, en amont et en aval, à un important travail d'information.

\section{Une grande préparation en amont}

Les contacts de plus en plus fréquents entre dirigeants, loin de réduire la charge de travail des diplomates ont au contraire pour effet de l'augmenter. Les rencontres, négociations et visites doivent être préparées, gérées et prolongées (informations préalables, mise en évidence des points d'accord et remontée des seuls points de désaccord, résolutions des "détails techniques » de plus en plus nombreux et importants). Les diplomates jouent en outre un rôle de synthèse politique visant à produire une position, à garder une cohérence, à construire un discours; d'où la répétition des éléments de langage (un point qui n'est pas repris dans un discours officiel pourrait être perçu comme le «signe » que le sujet n'est plus considéré comme important par le ministère) ${ }^{6}$. "Tous les propos tenus durant les visites officielles sont cadrés par des travaux préliminaires servant à déterminer les sujets à aborder et à écarter les développements indésirables. Ce travail de mise en forme permet d'isoler les thématiques, de les valoriser, en se libérant de tout ce qui ne rentre pas dans le ton de l'échange $)^{7}$. Au-delà d'une rencontre et d'un problème technique particulier, il s'agit de faire perdurer la relation bilatérale. La direction géographique, en lien avec l'ambassade française du pays visité, détermine la liste des thèmes à aborder et des personnes à rencontrer, reprise dans une note de cadrage. Des échanges préalables avec le cabinet du ministre et d'autres ministères éventuellement intéressés par la visite sont réalisés, afin de lister et de coordonner les différentes demandes éventuelles. Au sein de 1'ambassade, les conseillers techniques sont invités à rédiger à l'intention du chef de poste des fiches techniques sur les thèmes susceptibles d'être liés à la visite et qui l'aideront pour la rédaction des télégrammes diplomatiques et l'organisation de la visite. De même, l'ambassadeur et son équipe sont souvent amenés à rédiger les discours officiels qui seront prononcés par le responsable politique. La préparation et la négociation minutieuse des programmes, des thèmes abordés (et de ceux que 1'on souhaite éviter), des éléments logistiques, jusqu'à l'éventuel échange de cadeaux, ont pour objectif de limiter tous les imprévus et de désamorcer à l'avance tout risque de friction, toute tension ou malaise entre les personnalités ${ }^{8}$. L'objectif de la visite n'est pas tant de régler les problèmes en suspens, ce qui sera fait en amont ou en aval de la visite par les

\footnotetext{
${ }^{6}$ Iver B. Neumann, «A speech that the entire Ministry may stand for, or: Why diplomats never produce anything new », International Political Sociology, 2007, ${ }^{\circ} 1$, p. 183-200.

${ }^{7}$ Meredith Kingston de Leusse, Diplomate. Une sociologie des ambassadeurs, Paris, L'Harmattan, 1998, p. 149.

${ }^{8}$ Ibid.
} 
diplomates ou les experts concernés, mais d'afficher symboliquement une bonne entente, d'affirmer une volonté politique de coopération. Cela permet en outre d'augmenter la confiance et l'estime réciproque entre décideurs, donc de faciliter les relations à venir. Chaque rencontre ou chaque coup de téléphone entre dirigeants est l'occasion de la rédaction de documents pour le responsable politique français (président, ministre ou parlementaire) : biographie des homologues rencontrés, note de cadrage (ce qu'on attend de l'entretien), note d'entretien (on se renseigne à l'avance sur les sujets dont les responsables veulent parler). L'ancien diplomate britannique Carne Ross évoque avec une certaine ironie le cas de ministres qui, avant une rencontre avec leurs homologues, n'ont pas suffisamment travaillé les dossiers qui leur ont été préparés et sont incapables d'arriver à un accord précis ${ }^{9}$.

Les décideurs politiques ne pouvant être en permanence avisés de tous les problèmes et se concentrant sur les dossiers de crise, les diplomates doivent être à jour de chaque dossier potentiellement critique et développer une «attente active» (selon le terme de Neumann) pour être prêt quand surviendra une crise qui entraînera le déplacement d'un décideur ${ }^{10}$. L'influence des diplomates dépend donc de leur capacité à fournir au «bon moment» les éléments qui répondront aux enjeux tels que les perçoivent les politiques. La capacité à faire évoluer le cadre qui structure la définition même de ces enjeux est, quant à elle, plus limitée, du fait de l'existence de « lignes rouges », de grandes tendances dans les rapports entre États et des arbitrages entre divers intérêts tant internes qu'externes. Changer progressivement le cadre suppose un travail constant et continu. Un rédacteur précise : «On essaye d'irriguer en permanence des gens qui comptent en informations, en petites notes, en bullet points. Notre travail, c'est de jouer les rabat-joie. D'introduire de la complexité, de rappeler que les choses ne sont pas si simples. Mais la complexité, pour qu'elle ne soit pas mal prise par les décideurs qui ont des millions de choses à lire, il faut qu'elle soit exposée clairement dans des notes courtes et synthétiques. Si nous voulons être lus, il faut essayer de dire clairement des choses compliquées. » Il s'agit d'anticiper les problèmes pour avoir les éléments suffisants en cas de besoin ou pour prévenir les problèmes avant qu'ils ne se transforment en "crise ». "Le métier de diplomate, c'est un travail de démineur : voir quelles sont les différences de vue et quelles sont les positions communes pour expliquer à Paris quelles sont les raisons de divergences et voir comment amener les autorités locales à nos vues ou trouver une solution médiane », selon un conseiller politique en ambassade. Le diplomate "médiateur » doit intérioriser la posture politique afin de pouvoir innover tout en restant « dans la ligne », conforme aux positions définies collectivement. Le risque alors, pour être écouté des décideurs, est de ne présenter que les analyses et les solutions qui seront acceptables ou attendues par les décideurs politiques ${ }^{11}$.

Il y a également parfois une certaine concurrence avec les membres des cabinets ministériels (autres que celui du MAEE) qui peuvent avoir la tentation, pour garder le contrôle de leur domaine spécialisé, de négliger les informations apportées par les diplomates. Mais les diplomates ont la connaissance des réseaux, des personnes qui ont l'information adaptée pour l'action, pour arbitrer, faire la synthèse entre les positions différentes quand un ministre «technique » vient négocier un dossier relevant de son portefeuille, mais pouvant avoir des implications plus larges qu'il ne maîtrise pas forcément. Par des réunions préparatoires au MAEE avec les conseillers concernés, les diplomates participent largement à la redéfinition

\footnotetext{
${ }^{9}$ Carne Ross, The independant Diplomat : Dispatches from an Unaccountable Elite, Cornell University Press, 2007.

${ }^{10}$ Iver B. Neumann, « To be a Diplomat », International Studies Perspectives, Vol. 6, n ${ }^{\circ}$, February 2005, pp. 72-93.

11 Michael Barnett, «The UN Security Council, Indifference, and Genocide in Rwanda», Cultural Anthropology, 1997, nº, p.551-578.
} 
des problèmes, à la recherche des solutions adaptées, en lien avec les " agents traitants » des autres ministères.

\section{Accompagner et gérer les visites}

Ce travail, qui impose de lourdes contraintes (être au service des visiteurs, accepter de longs horaires y compris la nuit ou le week-end, etc.), est parfois dévalorisé, notamment par les diplomates qui ne sont pas issus de la carrière. George Ball, un haut fonctionnaire du Département d'État américain (ancien représentant auprès des Nations Unies) sous l'administration Kennedy, puis Johnson, à qui le président Carter proposait un poste d'ambassadeur dans une capitale prestigieuse l'aurait décliné en précisant qu'il ne voulait pas « devenir un tenancier d'hôtel pour membres du Congrès en visite ${ }^{12}$.

Evan G. Galbraith, quand il était ambassadeur des États-Unis à Paris s'est élevé contre ces visites d'officiels de différentes natures et a tenté d'en chiffrer le poids. " En 1983, 13.806 officiels américains sont passés par Paris. 844 étaient des officiels de haut niveau (secrétaires adjoints et au-dessus) qui imposaient à l'ambassade le travail d'au moins deux personnes par jour et par visiteur (calculé d'après le nombre d'heures de travail et l'effectif: par exemple une personne travaillant huit heures ou deux personnes travaillant quatre heures). [...] On arrive ainsi en 1983 à l'équivalent de 16 personnes travaillant à plein temps auprès des visiteurs officiels. Ce chiffre ne correspond pas seulement au personnel administratif. Une part importante du travail a été assurée par les fonctionnaires des sections politique et économique. Nous avons calculé que ces derniers consacraient aux visiteurs au moins $11 \%$ de leur temps, soit l'équivalent de deux fonctionnaires à temps plein. À titre de comparaison, nous avons établi que les fonctionnaires des services politiques et économiques passent $14 \%$ de leur temps à établir des rapports, une de leurs principales tâches $)^{13}$. Les diplomates que nous avons rencontrés, étaient très engagés dans cette activité dont l'importance (notamment en termes de reconnaissance du travail accompli par les responsables politiques) leur paraissait évidente.

Lors de nos observations, nous avons pu assister à la préparation de plusieurs visites : de parlementaires, de ministres et d'autres responsables politiques. Ce genre de visites, fréquentes dans des postes d'importance régionale comme ceux que nous avons étudiés, donne lieu à une préparation intense. Échanges de mails, de courriers, établissement de programmes progressivement réévalués et négociés (tant avec les autorités françaises qu'avec les autorités locales), voire, parfois, venue de «précurseurs» à l'ambassade. Ce type de visites est programmé plus ou moins à l'avance et peut être très dépendant de l'actualité politique. Même quand la visite est prévue longtemps à l'avance, les impondérables sont toujours nombreux. Les visiteurs sont souvent animés par la volonté de « rentabiliser » au maximum leurs déplacements : rencontrer le plus de personnalités importantes, faire des gestes symboliquement significatifs, avoir des retombées médiatiques, etc., ce qui est susceptible d'être renforcé par l'effet de concurrence au sein des cabinets ministériels. De plus, les visiteurs peuvent réagir aux propositions qui leurs sont faites, avoir de nouvelles idées, de nouvelles exigences, juste avant leur arrivée. Même si le programme est prêt depuis longtemps, ils ne s'y intéressent dans le détail bien souvent que quelques jours avant, étant eux aussi soumis à un rythme intense et à un travail réactif. D'autre part, les changements et incertitudes peuvent aussi venir des autorités locales, réputées mal organisées et imprévisibles dans certains pays en voie de développement. L'incertitude est d'ailleurs un moyen de

\footnotetext{
${ }^{12}$ Cité par J. Shaw, The Ambassador : Inside the Life of a Working Diplomat, New York, Capital Books, 2006.

${ }^{13}$ Evan G. Galbraith, Ambassadeur de choc, Paris, Stock, 1986, p. 124.
} 
pression dans les négociations politiques, développant une certaine appréhension par rapport aux imprévus, aux changements de programmes. Une fois la visite commencée, il est également nécessaire de bien gérer les différentes personnes qui la composent. Se plier aux désirs et exigences des visiteurs, même si on les désapprouve, est souvent nécessaire. Ce type de travail demande de s'adapter rapidement, de garder son calme et sa bonne humeur malgré tout, même si cela paraît loin des grandes questions politiques. Les moments d'attente ou les instants passés à régler de petits détails occupent le temps et l'énergie. Ce travail est accepté du fait de l'importance des détails, bien connue de la part des diplomates rencontrés, même $\mathrm{s}^{\prime}$ il peut devenir frustrant à la longue ou quand ces efforts ne sont pas reconnus.

Les visites les plus codifiées et qui demandent le plus de préparation sont les voyages présidentiels ${ }^{14}$. Il existe d'ailleurs trois niveaux de visite en fonction de l'importance protocolaire accordée à l'événement : la visite d'État, rare et limitée au maximum à une par pays, par septennat ou quinquennat, la visite officielle et la visite privée, peu médiatisée en principe et motivée par des raisons personnelles ou des négociations secrètes. Une visite officielle, et plus encore une visite d'État, doivent être programmées et préparées dans les moindres détails. C'est le chef du protocole du MAEE qui dirige les opérations. Une mission préparatoire en négocie tous les aspects, largement codifiés par le protocole pour la visite d'État, avec l'ambassade de France et le MAEE ou la présidence du pays visité. Elle se rend dans le pays pour vérifier sur place tous les arrangements (sécurité, horaires, déplacements, répétition des futures actions officielles, traque des imprévus...). Souvent, des travaux sont faits à la résidence pour recevoir le président, une partie des vivres et des vins peuvent être emmenés avec les nombreux invités (proches, journalistes, industriels, etc.) pour le repas traditionnellement offert en retour à l'ambassade de France par le président français à son homologue visité. La gradation entre différents niveaux de visite permet un subtil jeu d'affichage et de contrôle de la portée symbolique d'un déplacement. Ainsi, en 1986, le Premier ministre israélien Shimon Pérès se rend à Paris. L'ambassadeur français remarque : « À Jérusalem, on considère ce voyage comme revêtu d'un caractère très officiel. Tel n'est pas le point du vue du Quai d'Orsay qui n'estime pas nécessaire ma présence à Paris, puisqu'il s'agit d'une visite "privée". Mes interlocuteurs israéliens relèvent le caractère artificiel de la distinction que nous établissons. Pour eux, ce n'est pas tant la forme de 1'accueil qui compte que le contenu des conversations. Ils regrettent mon absence à des entretiens dont on me détaille soigneusement la liste: chef de l'État, Premier ministre, président de l'Assemblée nationale, ministre de l'Économie et des Finances ${ }^{15}$. Le diplomate est confronté à la difficile conciliation de messages divergents à destination d'auditoires spécifiques. À la suite de la visite, l'ambassadeur et/ou les autres diplomates qui ont accompagné le responsable politique en déplacement rédigent un télégramme diplomatique. Un point est fait éventuellement sur l'influence, auprès des autorités locales et dans les médias, de la visite. Quand le diplomate y a assisté, un compte rendu des échanges peut aussi être demandé, verbatim des échanges qui peut ou non être publié, mais servira de base par la suite aux futures relations et rencontres. Même quand un déplacement est annulé, il est nécessaire de gérer la portée symbolique de ce non-événement. C'est le cas, par exemple de la rencontre entre Gordon Brown et Nicolas Sarkozy de juin 2007 qui sera remplacée par une conférence téléphonique. L'ambassade a dû prendre en charge les conséquences du

\footnotetext{
${ }^{14}$ Leur nombre a augmenté avec chaque présidence : cinq voyages en moyenne par an pour le général de Gaulle, quatre par an pour Georges Pompidou, onze pour Valéry Giscard d'Estaing, quatorze pour François Mitterrand et vingt-sept pour Jacques Chirac, d'après Denis Fleurdorge, Les rituels et les représentations du pouvoir, Paris, Editions Zagros, 2005. Depuis l'arrivée du président Nicolas Sarkozy, le nombre de voyage a encore connu une augmentation considérable.

${ }_{15}$ Alain Pierret, Ambassadeur en Israël, 1986-1991, Paris, Desclée de Brouwer, collection «Midrash », 1999, p.33.
} 
changement dans le programme et expliquer inlassablement aux journalistes : «Non, cela ne traduit pas des problèmes entre les deux hommes, il n'y a pas de différend sur la politique européenne. C'est juste une question d'agenda surchargé ! ».

Les négociations bilatérales sont généralement mises en scène comme un contact direct entre les autorités politiques des deux pays; l'ambassade et l'administration centrale étant censées n'avoir qu'un rôle d'accompagnement et de préparation des délégations ministérielles. La science politique, les spécialistes des relations internationales et les médias dessinent un tableau des négociations où tout se jouerait dans les tractations entre dirigeants et pour lesquelles l'ambassadeur est essentiellement un intermédiaire. Il ne resterait qu'un ensemble de "petites» négociations (qui peuvent être parfois longues et fastidieuses), indispensables pour assurer le succès des visites politiques: convaincre des interlocuteurs locaux importants de consacrer de leur temps au visiteur officiel français, obtenir des autorités locales un bon accompagnement en matière de logistique et de sécurité, etc. Or, l'observation détaillée des activités des diplomates montre que ce travail en coulisses est central dans la construction de la relation. Beaucoup de grands accords internationaux, avant d'être signés par les dirigeants politiques ont été patiemment construits par un long travail en commission ou en groupes restreints entre diplomates des différents pays. Matthieu Osmont donne l'exemple de l'accord quadripartite sur Berlin, signé le 3 septembre 1971: "Cette négociation s'est jouée à différents niveaux : celui des ambassadeurs, mais aussi celui de leurs conseillers, qui se sont réunis entre eux à un niveau hiérarchique certes "inférieur" - mais qui a tout de même son importance, comme nous le verrons. Les ministres des Affaires étrangères, les directeurs d'Europe des différents ministères, les chefs d'Etat et de gouvernement sont également intervenus à certains moments-clés de la négociation. Enfin, les canaux officiels de la négociation ont coexisté avec des canaux "officieux" $\gg{ }^{16}$.

Plus généralement, l'existence de différentes logiques sectorielles (malgré le rôle centralisateur de 1'ambassadeur, ce que font les militaires en matières de coopérations militaire ou les initiatives des services de coopération économiques et culturelle, peuvent parfois relever d'autres dynamiques que les échanges politiques sur lesquels se concentrent la chancellerie), de différents intérêts (ce que certains qualifient "d'agendas multiples », y compris l'agenda médiatique de plus en plus prégnant), de différentes temporalités (comme celles opposant la patiente construction d'une relation bilatérale à l'urgence d'une réaction lors d'une prise d'otage ou d'une guerre civile), produit un jeu diplomatique complexe et multiforme. La multiplication des conflits, des enjeux, des arènes de discussion avec la fin de la guerre froide, la globalisation de l'économie et des sociétés, conduisent à faire de la négociation un processus de plus en plus éclaté et permanent. "Nos sociétés demeurent conflictuelles. Leur politisation croissante - c'est-à-dire l'exacerbation des concurrences entre les projets politiques et sociaux, sur fond d'autonomie et d'affirmation de soi des individus conduit à une multiplication des occasions et des motifs de conflits (donc de négociation) $)^{17}$. Le diplomate n'est peut-être plus le seul acteur en lice, mais son rôle de coordonnateur et d'organisateur s'en trouve renforcé tandis que son analyse et ses initiatives sont toujours nécessaires pour faire avance les dossiers qui ne sont pas sous les feux de l'actualité.

\footnotetext{
${ }^{16}$ Matthieu Osmont, «La négociation de l'accord quadripartite sur Berlin (1969-1971) : le rôle du groupe de Bonn », Relations internationales, $\mathrm{n}^{\circ} 135,2008$.

${ }_{17}$ Reynald Bourque et Christian Thuderoz, Sociologie de la négociation, Paris, La Découverte, collection «Repères », 2002, p. 114.
} 


\section{Le travail d'information et les technologies de l'information et de la communication (TIC)}

L'information est un renseignement qui apporte un surplus de connaissance censé être utile au destinataire. Dans le contexte de l'administration publique, est considérée comme " information » toute donnée pertinente dont la collecte, le traitement, l'interprétation et l'utilisation sont destinés à concourir à la réalisation d'une mission gouvernementale. Il ne s'agit donc pas seulement de faits bruts, de données purement factuelles, mais de faits sélectionnés, mis en forme et commentés.

\section{L'information diplomatique : entre analyse politique et traitement routinier}

Si d'autres groupes professionnels tels les journalistes ou les chercheurs produisent, diffusent et analysent des informations, les diplomates doivent veiller à produire une information d'un type particulier. Celle-ci est tout d'abord marquée par sa nature officielle. Représentant son pays à l'étranger, le diplomate, et notamment l'ambassadeur, est celui qui est légitimement mandaté pour collecter les informations auprès des autorités locales et les transmettre à son administration. Les messages auront donc une forme et un contenu pour une part dictés par les obligations qu'impose cette fonction. L'objectif est de participer à la production d'une certaine image publique d'une relation bilatérale ou de la gestion multilatérale d'un dossier. L'information produite par les diplomates doit également orienter les décisions et les choix politiques. Elle doit donc participer à la construction de la position de la France, des éléments de langage, du cadre d'action de la politique étrangère. Selon Jovan Kurbalija ${ }^{18}$, le traitement de 1'information en diplomatie repose sur trois grandes « techniques ». Tout d'abord la contextualisation. Une information n'a de sens que si elle est replacée dans son contexte. Elle prend sens par rapport à une situation locale (ce qui nécessite une connaissance fine du pays, des décideurs, du climat politique...). Elle s'inscrit dans la ligne du ministère, la politique étrangère globale, au niveau de l'ensemble des services diplomatiques auxquels appartient le rédacteur, ce qui nécessite une certaine transversalité entre les services. La deuxième technique évoquée est la " prospection d'information 》 (datamining) : l'objectif est de rassembler de grandes séries de données capables de donner du sens à une information ponctuelle. L'auteur cite l'exemple des " schémas de vote » que dresse le département d'État pour chaque pays à l'ONU : une prise de position particulière au Conseil de sécurité a plus de sens si on peut la rapprocher de toutes les décisions antérieures, sur des sujets similaires, du pays en question et de celles de groupes de pays. La troisième et dernière technique qu'il évoque est la synthèse: face à la prolifération d'informations de toutes natures, il est de plus en plus important d'en extraire les plus pertinentes. Il ne s'agit pas simplement de résumer un grand nombre de pages en quelques lignes, ce qui induit toujours un risque de perte d'information et de simplification, mais de penser le texte en fonction des niveaux de précision pertinents et de la nature des informations utiles pour chaque type d'usage qui en sera fait.

De plus, ces trois techniques s'inscrivent dans des modalités de travail variées. Jovan Kurbalija en distingue trois : tout d'abord les activités répétitives et routinières (vérification d'informations et d'antécédents lors de la délivrance d'un visa...). Ensuite les activités semirépétitives (synthèse et suivi de nombreuses conventions internationales sur le commerce,

\footnotetext{
${ }^{18}$ Jovan Kurbalija, « Knowledge Management in Diplomacy », Knowledge and Diplomacy, Ed. Jovan Kurbalija, DiploPublishing, 1999.
} 
l'environnement, etc.) : les conclusions de nombreuses réunions multilatérales ou bilatérales de suivi de tel ou tel accord doivent être résumées et diffusées de façon assez standardisée. Enfin, il y a, au cœur des négociations, des situations de crise, des activités non répétitives nécessitant un grand nombre d'informations hétérogènes qui ne peuvent en aucun cas être traitées de façon standardisée et doivent déboucher sur des analyses créatives capables de favoriser des décisions innovantes. Cette grille de lecture permet de problématiser un certain nombre de questions et d'observations apparues durant l'étude. En premier lieu, il est difficile de concilier un traitement standardisé et bureaucratique de l'information et le besoin, dans certaines situations, d'analyses plus personnalisées, plus créatives, en partie fondées sur une forme d'intuition, de perception difficile à formaliser («sentir la situation»). Certains diplomates, notamment de jeunes rédacteurs ou de jeunes conseillers, se plaignent d'être noyés sous des tâches semi-répétitives et de ne pas avoir la possibilité de passer à des analyses plus innovantes, de prendre le temps de réfléchir. Pour cela, ils réclament également du temps et des moyens pour des réunions transversales, des missions sur le terrain, des rendez-vous avec différents représentants du pays. En fait, la frontière entre les activités semi-répétitives et les activités répétitives n'est pas fixe, elle est susceptible de variations en fonction de l'actualité, des choix politiques opérés, des modes de gestion du travail des subalternes, etc. En second lieu, la division du travail et l'existence de tâches plus ou moins complexes induisent des enjeux de pouvoir et de prestige. Les formes de délégation du travail de traitement de l'information peuvent varier d'un pays à l'autre. La lecture de la littérature internationale et les entretiens auprès de diplomates français à propos des pratiques de leurs homologues étrangers montrent que par rapport à la France, les États-Unis et la Grande Bretagne pratiquaient une plus grande division et routinisation du travail de traitement de l'information avec, pour les sujets politiquement sensibles ou d'actualité, une répartition des tâches de recueil et d'analyse de données par un plus grand nombre d'agents avec une plus forte spécialisation technique. En France, le choix est fait d'une approche plus généraliste sur une base géographique (à l'exemple du rôle central du rédacteur et de sa sous-direction). Cela favorise une plus grande capacité de synthèse, dont la contrepartie est le risque de biais face à une trop grande masse d'informations et une difficulté de gestion des rapports hiérarchiques, la synthèse pouvant être faite à différents niveaux.

Ensuite, la création d'un système très dichotomique séparant de façon tranchée et conflictuelle «ceux qui pensent », le travail noble, et "ceux qui exécutent », alors que l'approche anglo-saxonne favorise plus la perception d'un continuum. Comme l'explique un rédacteur à l'issue d'une conférence téléphonique avec ses homologues de différents pays : " Je trouve qu'on a des cultures très différentes avec les Anglo-Saxons. Ils sont à fond dans la procédure, la rédaction de non-papiers, la mise en place de commissions, de réunions... Et nous, on déteste ça : tout cet ensemble de discussions sur les comités de suivi, la définition des suivis, les points à lister, etc. Ce ne sont pas les mêmes formats d'équipe également. A Washington, ils sont 30 à travailler sur ce pays, ici je suis tout seul. En France on est plus dilettante, on est plus dans l'initiative politique: lancer une idée, et les autres ont plus de moyens à mettre en œuvre dans le suivi. Quand il y a 30 personnes pour suivre un dossier, chacun veut proposer sa commission, son groupe de suivis, produire du papier et des rapports. Les Britanniques ont une grosse présence sur le terrain en ce qui concerne le renseignement militaire. La Norvège, elle, patronne l'aide humanitaire sur la région. Ils vont être au courant de tous les agendas. Le plus important pour moi, c'est d'être dans la liste de diffusion de tous ceux qui participent à ces conférences téléphoniques du mercredi et du jeudi. » Un de ses collègues précise : «Dans d'autres pays, c'est beaucoup plus pléthorique. Pour le Soudan, par exemple, le rédacteur qui s'en occupe, ainsi que du Darfour, est tout seul, alors que les Britanniques ont trois personnes et les Américains 10. Cela ne veut pas dire qu'un Français vaut trois Britanniques, mais c'est une façon différente de travailler. Sur les détails pratiques, 
ils sont mieux placés que nous. Par exemple, ils sont capables de produire une carte géographique actualisée avec tous les mouvements de population, les ONG, et tout un tas d'informations. Mais le fait qu'il n'y ait pas au Royaume-Uni une personne ressource unique fait qu'ils perdent en capacité d'analyse politique. ») Enfin, les agents C et B (à l'exception des responsables des systèmes d'information, protégés par leur «niche » technique) peuvent ainsi se sentir dévalorisés et exclus du travail le plus noble en étant consignés aux tâches les plus répétitives, alors qu'ils pourraient être associés à certaines phases du traitement de 1'information, notamment à la prospection de données. Une tâche semi-répétitive de «prospection » et de suivi de donné peut jouer un rôle important. Par exemple, dans une Représentation permanente, une secrétaire (agent C) s'était vu confier un travail de gestion de dossier, permettant de mettre à jour des dossiers et de mieux défendre la position française, qui avait à ses yeux rendu son travail plus riche et intéressant: "Ce qui a changé depuis 1'arrivée de la conseillère, c'est un gros travail qu'elle m'a confié grâce auquel je peux vraiment participer au dossier des droits de 1'homme. C'est un travail de gestion et de suivi des dossiers. Quand les gens portent plainte contre la France et que la France est condamnée, généralement elle paye en retard. Mais il arrive souvent que la France ait payé, sans que cela ait été retiré de l'ordre du jour. C'est-à-dire que le Conseil de 1'Europe continue à demander un paiement qui a déjà été fait. Donc, mon travail consiste à recenser les affaires dans lesquelles la France a été condamnée. Quand la France est en retard pour payer, la conseillère fait une note auprès de la direction juridique. Quand la France a payé et que l'affaire n'a pas été retirée de l'ordre du jour, je signale cette anomalie afin que la conseillère puisse en demander le retrait. Si la gestion du suivi des affaires est bien faite, cela permet d'éviter qu'il y ait des pressions indues sur la France concernant des affaires qui ont été réglées, et cela renforce l'image de la France qui était ternie par cette question des retards. Grâce à mon travail et à celui de la conseillère, la France a pu améliorer un peu sa réputation de mauvais payeur. Quand la conseillère est absente, j'ai le contact avec la direction juridique à laquelle je transmets directement mes données. Je connais la personne, en général cela se passe bien. »)

\section{Des TIC qui ont encore peu modifié le contenu et la division du travail}

Le système standardisé et stabilisé de communication diplomatique serait aujourd'hui remis en cause. D'après le rapport Tavernier : «Dans l'exercice de sa mission d'information, 1'ambassadeur se trouve concurrencé par les médias. Bénéficiant de moyens matériels et d'un accès privilégié aux informations, certains journalistes exercent par leur audience une influence directe sur la décision politique. Les journalistes de l'Agence France Presse à Washington sont plus nombreux que les diplomates de l'ambassade de France. Les grandes entreprises mènent leurs projets, non plus au rythme des télégrammes, mais à coup de courriers électroniques et de déplacements de leurs présidents pour rencontrer des chefs d'État sans que les ambassadeurs n'en soient toujours préalablement informés, alors même que le président de la République ne manque pas d'emmener des chefs d'entreprise dans ses déplacements officiels ${ }^{19}$. L'observation du travail des diplomates montre pourtant que les activités de rédaction de note de synthèse et de télégrammes restent des moments forts, auxquels soin et attention sont apportés. Comme par le passé, la volonté la plus fréquente est de produire un grand nombre de télégrammes qui seront lus et considérés comme importants, ou, à tout le moins, de rédiger et signaler un maximum d'informations de manière à ne pas pouvoir être pris en défaut à l'avenir pour ne pas avoir transmis tel ou tel élément qui se révèlera important a posteriori. À tel point que les différents secrétaires généraux du Quai

\footnotetext{
${ }^{19}$ Rapport d'information déposé le 20 février 2002 « sur le réseau diplomatique et le rôle des ambassadeurs » et présenté par Yves Tavernier, député.
} 
d'Orsay envoient régulièrement à l'ensemble du réseau des instructions demandant de réduire le nombre de télégrammes, leur longueur et, parmi eux, la proportion d' «urgents » ou de « confidentiels ».Toutefois, les mails, les listes de diffusions, les recherches sur Internet permettent à la fois d'élargir et d'accélérer le processus de traitement de l'information. Les formes plus traditionnelles (lecture et rédaction des télégrammes et des notes de synthèse, de la presse) occupent toujours une part importante du travail des diplomates, mais s'y ajoutent d'autres sources d'informations, des échanges plus informels de données et d'analyse. De plus, les informations, ou du moins une partie d'entre elles, semblent de plus en plus rapidement obsolètes et dépassées.

L'observation d'une journée de travail d'un conseiller dans une ambassade ou d'un rédacteur à Paris montre bien les conséquences de cet élargissement et de cette accélération du traitement de l'information. Ce qui frappe l'observateur, en comparaison d'autres activités de travail, c'est la fréquence de l'interruption et du changement de tâche en cours de séquence d'activité sans que celle-ci ne résulte nécessairement d'une contrainte extérieure. La sociologie du travail et l'ergonomie ont largement insisté sur la surcharge cognitive qui résultait des interruptions de tâche subies, par exemple, pour un cadre dérangé par un subordonné lors de la rédaction d'un document ou une infirmière qui doit interrompre un soin à l'appel d'une collègue. Si les diplomates ont aussi à subir ce type d'interruptions intempestives (coup de téléphone, demande urgente d'un supérieur, cas complexe soumis par un subordonné, etc.), un certain nombre d'autres interruptions semblaient volontaires et spontanées : au milieu de la lecture de sa collection de télégrammes du matin, le diplomate s'arrête pour commencer la rédaction d'une note, puis, avant d'avoir terminé celle-ci, va consulter sur Internet des sites d'information ou des sites spécialisés sur les sujets ou les pays de son portefeuille, tout en jetant, dans certains postes, un œil distrait sur la chaîne d'information continue d'une télévision allumée en permanence avec le son coupé, avant de reprendre la lecture des télégrammes. Seules les tâches les plus délicates et les plus formelles, comme la rédaction d'un télégramme diplomatique, sont réalisées en continu. Ce comportement, qui semble disperser inutilement le travail et les efforts, s'explique tout d'abord par la volonté d'avoir le plus rapidement possible l'information, de ne pas passer à côté d'une nouvelle importante. Les diplomates de rang plus élevé peuvent parfois déléguer ce travail de veille à des subordonnés. Autre explication : casser la routine et garder un rythme de travail rapide sur une longue journée de travail. Une dernière justification, moins souvent avancée, serait que cette forme de fragmentation de l'activité pourrait finalement faciliter l'intégration, par le diplomate, d'informations d'origine très variées et hétérogènes en une vision relativement unifiée. Le risque est toutefois de se perdre dans le flot de nouvelles et de céder à l'attrait de l'immédiateté, à la recherche du scoop, de l'information inédite qui conduit à se connecter et à surfer de trop longs moments. Certains diplomates ont alors le sentiment d'être «scotché » à l'actualité, le «nez dans le guidon», sans possibilité de recul ou de réflexion. Sentiment qui peut sembler paradoxal dans la mesure où il est en partie généré par les méthodes de travail mises en œuvre. D'autant plus que la médiatisation de certains dossiers conduit les pouvoirs publics à agir dans l'urgence. Les diplomates sont alors confrontés à un dilemme: soit tenter d'apporter une information sans avoir forcément le temps de prendre suffisamment de recul, soit prendre le temps d'une synthèse plus réfléchie, mais avec le risque que les décideurs n'attendent pas et prennent leurs décisions avec d'autres éléments. Bien sûr, la solution à ce problème est à chercher dans une démarche d'anticipation des problèmes et des crises qui pourraient éventuellement se poser («attente active »).

Internet met à disposition un flot croissant de données à un rythme de plus en rapide. Les sites des médias, des institutions publiques, des ONG, des entreprises, des think tank, des blogs de journalistes, de chercheurs ou d'opposants politiques, etc., peuvent apporter des informations intéressantes. Mais on y trouve aussi des analyses biaisées ou erronées, des 
répétitions inutiles. Comment évaluer la fiabilité et l'intérêt d'une source? Quels sites consulter sur tel ou tel sujet? Auprès de qui et comment confirmer et interpréter une information trouvée sur un site? Par exemple, un diplomate doit appeler un correspondant de l'administration du pays où il est en poste pour comprendre les raisons de la retranscription tronquée d'une rencontre bilatérale sur les sites officiels. Comment replacer et synthétiser l'information dans un contexte plus large, lui donner un sens pertinent pour l'action ? Tout cela demande l'expérience d'un secteur, la connaissance des personnes et donc, la constitution d'un réseau dans lequel un minimum de confiance a été établi, ce qui nécessite des rencontres de face à face, un système de don et de contre-don (échange d'information, invitations à déjeuner, services réciproques...) pour créer des liens. Au sein du MAEE, l'utilisation de l'Intranet, des mails, des listes de diffusion permet une diffusion et un partage plus rapides de l'information, moins protocolaires et moins hiérarchiques (un rédacteur peut ainsi correspondre régulièrement avec le conseiller du ministre sans passer au préalable par son supérieur). Encore faut-il que les supérieurs acceptent de déléguer à leurs subordonnés, ce qui n'est pas fait de la même façon dans toutes les directions. De plus, la capacité à prendre du recul, à contextualiser ou à relativiser une information dépend pour une part de l'ancienneté et de l'expérience accumulée, des savoirs-faire intériorisés.

Par ailleurs, si l'utilisation croissante des nouveaux moyens de communication permet, à condition qu'elle soit tolérée par la hiérarchie, certaines formes de démocratisation dans la diffusion de l'information et la participation au processus de décision, elle peut aussi engendrer de nouvelles inégalités. Le réseau fonctionne sur la base d'une certaine confiance en s'appuyant sur les relations personnelles. Un rédacteur (direction géographique) explique : «Par exemple, sur un dossier transversal comme celui-ci, il y a une multiplication de sources à solliciter, les dossiers sur l'Intranet, les archives, des sollicitations directes de personnes. Au final, j'ai plutôt l'impression de créer quelque chose, de participer à quelque chose qui est le fruit d'une contribution collective de nombreux services. Il y a aussi un esprit solidaire avec ta promotion. Ce sont les premiers qu'on sollicite. On est dans le registre de la sollicitation, il n'y a pas de système d'échange d'informations mais des dons d'informations qui sont ensuite retravaillées. Naturellement, c'est à la fois bien et bancal, car c'est basé sur le principe de la confiance faite au service donneur. Parfois c'est délicat, parce qu'on ne sait pas comment fonctionnent les autres directions géographiques ou politiques. On ne sait pas si elles retravaillent ou pas tes informations, s'il y a un phénomène de transformation. " Tout le monde ne sera donc pas automatiquement intégré dans les listes de diffusion et cela peut favoriser la constitution de réseaux informels fondés sur des catégories (d'âge, de concours, etc.) et l'exclusion de certains. L'absence de tradition de travail en équipe, d'accueil, de présentation et de formation des nouveaux arrivants fait que la constitution de réseau dépend largement d'initiatives individuelles. Pris sous la masse des commandes semi-routinières (notes, réponses à des demandes d'information, suivi de dossiers, lecture des télégrammes diplomatiques, du courrier et des mails), certains rédacteurs ont le sentiment d'être enfermés dans leur bureau, sans contacts avec leurs collègues. L'outil informatique, en multipliant les informations et les messages à traiter apparaît alors plus comme une source d'enfermement que d'ouverture. Seules des initiatives venues de la hiérarchie visant à faire travailler de façon transversale des rédacteurs de différents services (en les exemptant provisoirement de leurs obligations habituelles) peuvent aider ces agents à élargir leurs réseaux grâce à des moments et des lieux favorisant les contacts directs. Comme le déplore une rédactrice (direction géographique) : "Quand vos supérieurs ne vous donnent pas des consignes claires, quand vos collègues ne peuvent pas vous renseigner, quand vous passez des journées entières à ne discuter qu'avec des gens qui n'ont pas grand-chose à voir avec votre travail, que systématiquement on n'est jamais réuni alors qu'on fait partie de la même sous-direction et qu'on met deux mois à connaître les noms des uns et des autres, il est évident que 
l'information ne circule pas. Que les documents qui pourraient être partagés par tout le monde ne circulent pas, qu'il faut que chacun aille à la pêche, il est clair qu'il y a une perte d'efficacité... »

Au-delà de quelques réseaux ponctuels et informels, fondés bien souvent sur une origine commune (les énarques, les «Orients », les anciens de tel stage de formation, etc.), beaucoup de diplomates trouvent les solidarités collectives encore insuffisantes. Les moyens techniques offerts par l'Intranet ne suffisent pas à changer les comportements. Une rédactrice (direction géographique) remarque: "On n'est pas plus intelligent parce qu'on ne partage pas l'information, parce qu'on parle moins aux autres. Il y a des gens qui sont moins gradés que vous qui ont des choses intéressantes à vous dire, on peut parler à d'autres catégories que des catégories A. Peut-être qu'il faudrait d'ailleurs faire des stages mixtes, pourquoi faut-il que les A ne soient qu'avec les $\mathrm{A}$. Les $\mathrm{B}$ ont un cerveau, les $\mathrm{C}$ aussi... Il y a des $\mathrm{C}$ très brillants ici chez nous, d'autant plus maintenant qu'il y a des C, et des B a fortiori; il y a des C qui rentrent avec des niveaux de diplôme très comparables à ceux des A. Quand on voit quelqu'un qui arrive de DEA et qui est là, on peut vraiment lui parler ». De plus, si certains rédacteurs - mais pas tous - peuvent parfois voir leur rôle revalorisé, les agents de catégorie B et $\mathrm{C}$ qui participaient au traitement plus routinier de l'information (tris des courriers, des télégrammes diplomatiques, coupures de presse, recherches documentaires sur demande, etc.) peuvent voir leur rôle un peu plus marginalisé par l'usage des mails et des recherches directes sur le Net. Pour les télégrammes diplomatiques, le logiciel "Schuman », un système d'attribution automatique, va reporter la tâche du tri sur les rédacteurs, comme le remarque cette agent B : «Les télégrammes diplomatiques (TD) nous arrivent par le chiffre. Les agents vont les chercher au sous-sol, 4 fois par jour. Nous procédons par tri. Nous sommes la direction géographique qui a le plus de TD (36 pays plus la Représentation permanente à Bruxelles. C'est frustrant d'aller chercher des piles de TD. C'est une pile de $20 \mathrm{~cm}$ et de 300 TD par jour. C'est ingrat car mécanique. Il faut les trier et les classer selon les préférences de la direction. Par exemple on prend la collection 3, on lit chaque TD et on regarde quel rédacteur doit avoir tel TD, même si ça ne lui est pas adressé. Les agents doivent lire les TD et connaître les attributions de chaque rédacteur. La collection suit son cours. La sous-directrice vérifie les attributions. Une collection, c'est un jeu par sous-direction et quatre fois par jour. Cela fait 20 paquets à trier chaque jour. C'est un travail ingrat parce que ça n'appelle pas de réflexion, à part pour l'attribution. On est dans une situation particulière : le système Schuman est en déploiement, mais on continue de trier les TD... Avec le système, ils pensent se dédouaner de ce tri, mais en fait les rédacteurs, avec Schuman, seront obligés de faire ce tri. Ça leur donnera un peu plus de travail. Nous, on les aide énormément, en leur attribuant les TD : ils ont une bonne source de travail ». À Paris, les agents de catégorie C sont cantonnés aux tâches les plus routinières et les moins qualifiées du traitement de l'information, même si celles-ci peuvent avoir leur importance dans la production de la direction. C'est ce qu'explique une sous-directrice dans une direction géographique: «Ici, c'est une armée mexicaine! Il n'y a que des catégories A. On a aussi un pôle secrétariat pour toute la direction, avec deux agentes $\mathrm{C}$ qui sont administrativement rattachées à la sous-direction. Elles ne font pas de dactylo. Leur travail, c'est avant tout un filtrage des communications téléphoniques et de circulation de l'information: porter des plis dans d'autres services, photocopier les dossiers. La photocopie, ça peut être un gros travail quand il y a un dossier de visite, pour les visites officielles. C'est moi qui coordonne la rédaction du dossier et ce sont les secrétaires qui font les copies et l'assemblage. »

La dichotomie entre le travail noble d'analyse politique et les tâches moins valorisées de gestion et d'administration se manifeste par le fait que les informations qui sont le plus facilement partagées à travers les nouveaux outils de communication sont celles qui sont l'objet d'enjeux moins importants, d'un prestige plus faible. Dans les directions logistiques, 
qui ne traitent pas directement la matière noble, l'analyse politique, la délégation du travail intellectuel semble ainsi plus concevable. Un rédacteur (direction des Affaires Financières) remarque: "J'ai la chance d'avoir une secrétaire, ce qui n'est pas le cas de tout le monde, donc je lui délègue des choses avec un contrôle a posteriori ou avant avec prise de consignes. Je délègue beaucoup de choses parce que je n'ai pas forcément beaucoup de temps dans une journée, c'est-à-dire qu'elle me filtre mon courrier, elle me fait des petits points. Je suis aussi en train de la mettre au courant sur un certain nombre de dossiers à connaître. Sinon, je travaille beaucoup en réseau, c'est une chose au Quai d'Orsay qui n'est pas très développée encore... Je ne parle pas du réseau mail, je parle du réseau partagé, avec des fichiers partagés, avec un système de notes de corrections ou de modifications avec l'administrateur, mes collaborateurs, etc. Mais on est encore loin de travailler de manière complètement efficace ». De même, le seul cas connu de constitution d'un réseau d'échange d'informations et de connaissances techniques concerne le groupe des secrétaires généraux d'ambassade. Un chef de SAF (service administratif et financier, qui coordonne la gestion du poste diplomatique) explique : « J'ai créé un réseau par messagerie d'une trentaine de secrétaires généraux dans le monde. On était trente à prendre notre service en même temps, à la suite d'un stage de formation, au moment de la LOLF et la prise de responsabilité sur de nouveaux secteurs. On se donne des coups de main. Quand une personne a un problème ou une question, il y en a toujours un pour trouver une réponse, une solution. Ça s'est su et on nous a demandés d'étendre ça à l'ensemble du ministère. Mais il nous a semblé qu'un blog pour 150 pays, ça serait ingérable. On est trente à se connaître par le stage, je pense que si on ouvre ça à tout le monde, il risque d'y avoir une démobilisation. Par contre, on a accepté de se lancer dans une démarche de partage d'information. Jeudi prochain, on est quatre du réseau à venir rencontrer des personnes en cours de formation pour leur apporter un retour d'expérience de notre réseau de 30 personnes. " Là encore, on retrouve l'importance de la formation partagée dans la construction de réseaux de solidarité et de partage d'information, mais il s'agit d'un type d'information différent et perçu comme moins valorisant par les diplomates.

L'usage d'Internet offre donc de nouvelles potentialités et pourrait rendre possible de nouvelles façons de travailler, mais globalement, la logique de la "diplomatie traditionnelle » valorisant l'analyse politique reste très forte. Mettre l'information en perspective, lui donner du sens, vérifier les sources, éviter les redondances, transmettre des analyses utiles, nécessite toujours un savoir-faire, fruit de l'expérience, qui contribue à forger l'intuition du diplomate. Sur ce plan, les choses ont peu évolué. Voici, par exemple, la façon dont un ancien ambassadeur décrivait son travail comme jeune conseiller à Moscou entre 1956 et 1959 : «Un matin de juin, lisant dans notre Pravda quotidienne la liste de membres du Politburo ayant la veille assisté à un spectacle du Bolchoï, nous constatâmes, d'une part, que les noms de ces personnages manquaient, d'autre part, que le délégué Pospelov, jusque-là membre suppléant, figurait maintenant, selon l'ordre alphabétique en usage, parmi les membres pleins. On pouvait en conclure qu'un remaniement capital avait eu lieu. Mais le devait-on? Nous tentâmes de convaincre l'ambassadeur de télégraphier à Paris le fait, au moins, à titre d'hypothèse. Il s'y refusa énergiquement, estimant la base trop fragile pour en tirer une conclusion aussi énorme. Il manqua ainsi l'un des scoops de sa vie ${ }^{20}$. Aujourd'hui, cette intuition, cette capacité à lire entre les lignes et à trouver l'information pertinente est toujours d'actualité. Ce qui a changé c'est que l'on est passé d'une situation de pénurie d'information à une situation, bien souvent (à l'exception peut être de certains pays comme la Corée du Nord ou la Birmanie) de profusion. Le savoir-faire, l'expérience pour "sentir » ce qui sera le plus important, les « réseaux relationnels » pour corroborer l'information, le travail collectif de traitement et de mise en forme sont donc plus que jamais nécessaires.

\footnotetext{
${ }^{20}$ Henri Froment-Meurice, Vu du quai 1945-1983. Pour une Histoire XXe Siècle, Paris Fayard, 1998, p. 175.
} 
Notre enquête s'étant déroulée entre 2006 et 2008, elle ne nous permet pas de mesurer une éventuelle "transformation », sur cette courte période, du travail diplomatique. Il nous manque le recul de données longitudinales et homogènes sur la longue durée. Toutefois, la lecture des travaux des historiens de la diplomatie comme des autobiographies d'ambassadeurs suggère que les grands traits du métier ont peu changé malgré les bouleversements des relations internationales et les innovations techniques. Il existe toujours un ensemble de tâches, de savoir-faire à mettre en œuvre, d'analyses politiques à produire et à mobiliser pour l'action publique et la représentation de la France qui fondent la spécificité du travail diplomatique. Toutefois, ce travail reste peu connu et difficile à évaluer ou à «mesurer». Les diplomates que nous avons rencontrés réfléchissent beaucoup à leurs pratiques, mais cette réflexion n'est pratiquement jamais rendue publique, discutée ni partagée... Elle n'est donc pas «capitalisée », ne peut être cumulative. Il en résulte une double difficulté : en interne, cela rend plus difficile la transmission des compétences, mais aussi la reconnaissance de ce qui est produit, la valorisation des efforts fournis. Et pour les observateurs extérieurs, le rôle des diplomates reste mal connu. On dénonce systématiquement ce qu'ils coûtent au contribuable à défaut de savoir ce qu'ils font, ce qu'ils " produisent ». Au-delà des aspects les plus ostentatoires de l'activité, la représentation de la France et de ses intérêts à l'étranger et l'activité consulaire, le travail des diplomates est en définitive, pour l'essentiel, un travail « invisible ».

Depuis une quinzaine d'années, le sentiment d'urgence d'une réforme de la diplomatie s'est accru, notamment pour des raisons budgétaires. Le MAEE a en effet dû faire face, dans un contexte de restrictions budgétaires à des obligations croissantes (explosion du nombre de demandeurs de visas, par exemple). La question des moyens et de leur gestion est ainsi devenue centrale alors qu'elle était auparavant considérée comme secondaire. Une approche plus gestionnaire de l'organisation est défendue. La fusion en 1999 du MAE avec le ministère de la Coopération a fait entrer au ministère des cadres ayant une culture de l'action logistique et de la gestion de programme qui ont pu se faire les porte-parole d'une approche plus managériale. Cette dernière s'est trouvée légitimée par les réformes mises en œuvre dans d'autres pays, comme le Royaume-Uni où un vaste plan de modernisation, initié au Foreign and Commonwealth Office (FCO), a introduit un système de gestion des compétences inspiré des principes du monde industriel. Venus de la réforme plus générale de l'Etat, deux dispositifs de modernisation ont touché, "par le haut », le ministère : le plan de modernisation et la LOLF. En 2006, le MAE est le premier ministère à signer un contrat triennal de modernisation avec le ministère des Finances. Ce contrat prévoit 16 accords sur les réformes structurelles comme la gestion prévisionnelle des ressources humaines, la dynamisation de la gestion de l'encadrement supérieur du ministère, la rationalisation du travail consulaire, etc. Un objectif important est par exemple de réduire le nombre d'ordonnateurs secondaires dans les postes pour rationaliser la gestion et faire des économies d'échelle. L'accord prévoit en outre une baisse de 3,34\% des effectifs (ETP). Dans ce cadre, le Comité interministériel des moyens de l'État à l'étranger (CIMEE) a adopté une directive nationale d'orientation des ambassades (DNO), qui a pour objectif de proposer une typologie des postes diplomatiques en fonction des enjeux politiques et d'allouer les moyens humains, financiers et techniques en conséquence. La LOLF comporte deux volets distincts mais articulés, le volet " comptable » et le volet « contrôle de gestion ». Le volet comptable consiste à mettre en œuvre une nouvelle nomenclature comptable et donc budgétaire, de façon à imputer les coûts de fonctionnement sur des " programmes » (par exemple « Solidarité à l'égard des pays en voie de développement » ou " action de la France dans 1'Europe et dans le monde »). Le responsable de programme, en accord avec son ministre, définit une stratégie pluriannuelle et est responsable de son application. Chaque programme est décliné en objectifs, eux-mêmes 
mesurés par des indicateurs. C'est là le volet « contrôle de gestion » qui a pour objectif la mesure d'une performance de l'activité diplomatique. Par exemple le programme 105, " Action de la France dans l'Europe et dans le monde », a pour premier objectif de " défendre et représenter les intérêts de la France ». Les résultats sont mesurés grâce à différents indicateurs : le nombre de consultations du site Internet du ministère et des postes ainsi que la présence des français et de la langue française dans les institutions internationales. D'autres indicateurs portent sur le nombre de crises grave ou le nombre de négociations importantes au cours desquelles la position de la France a été renforcée. Tous ces indicateurs font 1'objet d'une vérification par le Parlement.

La mise en place de la LOLF implique, pour l'administration centrale, que les cadres du MAEE puissent rendre compte de leur activité ou de celle de leurs subordonnés à partir d'une quantification des résultats atteints (nombre de contacts, de documents produits, etc.). Elle suppose donc de préciser, dans chaque cas, quels sont les résultats attendus de l'action publique et les indicateurs permettant de les « mesurer ». Cela ne pourra être fait qu'à travers des indicateurs fins, plus qualitatifs que quantitatifs, nécessitant une bonne connaissance du métier et des activités des diplomates. Le langage des diplomates est plus celui de la stratégie et de la politique que celui de la gestion et de la comptabilité. 